\title{
LA ENSEÑANZA DE LA SOCIOLOGIA DE LA MEDICINA EN EUROPA Y USA *
}

\author{
Lisbeth M. Claus \\ (St Louis University)
}

\author{
Yvo Nuyens \\ (Universidad de Lovaina)
}

El objetivo de este artículo es doble. Primero, dar una idea general del status actual de la enseñanza de la sociología de la medicina, especialmente en los países europeos. Segundo, $: 1$ presentar algunos problemas referidos a los programas de educación de sociología de la medicina para estudiantes de ciencias sociales y sus requisitos didácticos $\mathrm{y}$ profesionales. En ambos enfoques se presenta la sociología de la medicina en el matco de la sociología (como disciplina y como profesión).

* Una primera versión en inglés de este artículo fue presentado en el Octavo Congreso Mundial de Sociología (Toronto, 18-24 agosto, 1974). La presente traducción es de Jesús $M$. de Miguel. Los autores agradecen también la amable cola. boración de E. Cassee (Holanda), C. Herzlich (Francia), E. Holst (Dinamarca), M Jefferys (Reino Unido) y M. Pflanz (Alemania Federal). 



\section{Origen y desarrollo de la disciplina y la profesión}

El desarrollo de la sociología de la medicina y la dirección de las investigaciones en este campo ajoyan la hipótesis de que la labor intelectual y científica responde a (si es que no está determinada por) la estructura del orden social. Como sucede con la estructura y organización de los servicios sanitarios, también los intereses actuales de los sociólogos de la medicina reflejan las características sociales, políticas y económicas de la comunidad. ${ }^{.}$Sin embargo, los desarrollos científicos, organizativos y tecnológicos en medicina $\mathrm{y}$ en servicios sanitarios han servido a lo que Margot Jefferys ha definido como la simbiosis de la medicina y la sociología.

La evolución desde un modelo etiológico de enfermedades infecciosas a un modelo multicausal de enfermedades crónicas ha contribuido notablemente a que los médicos ( $\mathrm{y}$ otras profesiones sanitarias) se hayan dado cuenta de las relaciones crecientes entre la biología y las ciencias sociales. El cambio de medicina curativa a preventiva ha puesto de relieve el problema de la diferente cooperación y participación de los miembros de la comunidad en programas de sanidad y ha confirmado la importancia de los factores sociales e interpersonales en la organización y utilización de los servicios sanitarios. Bajo la influencia de la psiquiatría y la medicina familiar se ha desarrollado el cuidado y enseñanza médicas globales. Como consecuencia, los programas médicos empezaron a incluir las ciencias humanas, entre las que se destaca la sociología de la medicina. Aunque hasta nuy recientemente las autoridades públicas tenían relaciones mínimas con el sector sanitatio, en estos últimos años se han incrementado considerablemente sus funciones sanitarias, fundamentalmente en temas de ordenación, control, planificación y fi-

1. Howard E. Freeman, Sol Levine y Leo G. Reeder, Handbook of Medical Sociology (Englewood Cliffs: Prentice Hall, 1972) 2a. edición, pp. 501-522. 
nanciación. Como una consecuencia lógica se ha producido un mayor énfasis en la utilización de la ciencia social en el desarrollo de políticas sociales y en la realización de programas de acción social.

Todas estas tendencias actuales han afectado al status de la sociología de la medicina en general y al papel del sociólogo de la medicina en particular. La década de los sesenta presenció un incremento espectacular de la sociología de la medicina, especialmente en los Estados Unidos, aunque desarrollos similares pueden ser observados en muchos'otros países. Las facultades de medicina y las escuelas de estomatología, sanidad y enfermeras han incluido a sociólogos en el cuadro de sus profesores, y algunas de ellas han desarrollado departamentos separados de ciencias humanas y de conducta, 0 institutos de ciencias sociales relativamente autónomos. Los sociólogos se han visto implicados en temas de educación médica, primero en los Estados Unidos, y posteriormente en los años setenta en países como Gran Bretaña, Holanda, Alemania (oriental y occidental), los países escandinavos y Bélgica. En algunos de estos países la investigación de sociología de la medicina ha obtenido una prioridad absoluta por parte de las autoridades públicas y académicas. En consecuencia, hay cada vez un mayor número de sociólogos e investigadores en el campo sanitario. Hay también una tendencia a atraer sociólogos a los procesos de creación de políticas sanitarias a nivel local y nacional. La sección de sociología de la medicina de la American Medical Association se ha convertido en la sección más numerosa que se dedica a un área sustantiva. Se observa que cada vez se publican un número mayor de monografías sobre sanidad en las revistas existentes, y que han aparecido otras revistas especializadas, como el «Journal of Health and Social Behavior» y «Social Science and Medicine». En comparación con los años sesenta no es necesario en la actualidad convencer a las personas de la importancia y el potencial de la investigación social en el cuidado sanitario, ni hay que estar preocupados con el futuro crecimiento y desarrollo en este campo. ${ }^{2}$

Hans Mauksch en un trabajo reciente ${ }^{3}$ distingue diversas etapas en el desarrollo de la sociología de la medicina en los Estados Unidos. Las siguientes no pueden considerarse en forma aislada, sino que deben situarse dentro del contexto de la sociedad:

(a) Investigaciones sobre temas sanitarios como estudios incidentales. Estas son principalmente contribuciones de individuos interesados

2. Idem.

3. Hans Mauksch, «The current status of medical sociology training in the USA», Conference on the Sociology of Medicine (Varsovia, 20-26 agosto 1973). 
en incrementar el conocimiento sobre estos temas con el puro propósito de hacer avanzar la ciencia, o con el deseo de promover en el público el deseo de la consecución de reformas sanitarias. Estas contribuciones se encuentran fundamentalmente en el campo de la sociología de la medicina.

(b) El papel de ciertos individuos, universidades y fundaciones. Mauksch señala aquí la influencia en el desarrollo de la sociología de la medicina de prestigiosos sociólogos como Parsons, Merton, Kendall, Bloom y Fox; el papel de universidades famosas como Columbia, Chicago y Yale, y finalmente la contribución de fundaciones como la Rusell Sage, Milbank y la Commonwealth Fund.

(c) La emergencia de la sociología de la medicina como un campo específico. La mayoría de los observadores coinciden en el impacto de la creación de la sección de sociología de la medicina dentro de la American Sociological Association en 1959 como la institucionalización y profesionalización de la sociología de la medicina en el mundo académicocientífico y en el mundo profesional-social.

(d) Emergencia de programas específicos de especialización. Desde los años setenta, en los Estados Unidos se han incrementado sensiblemente el número de cursos en sociología de la medicina al nivel de estudios no graduados, y se han desarrollado varios programas de especialización en sociología de la medicina al nivel de estudiantes graduados y estudios postdoctorales. Como puede verse en Mauksch ${ }^{4}$ y Hollingshead, actualmente la mayor parte de los departamentos graduados en Estados Unidos ofrecen cursos o especialización en sociología de la medicina.

Este breve resumen de las etapas del desarrollo de la sociología de la medicina en los Estados Unidos indica que el establecimiento de programas formalizados de especialización en este campo no es meramente un asunto académico, sino que debe ser situado en un contexto social, político y económico más amplio. Por otra parte, hay que llamar la atención sobre el hecho de que el nivel de especialización y profesionalización de una disciplina depende en buena medida de su preparación educativa. Por lo tanto, se espera un interés más intenso por parte de la profesión sociológica en la preparación de científicos en sociología de la medicina. Sin embargo, cualquiera que esté al tanto del mundo académico internacional (como se expresa en congresos, simposia, revistas profesionales, publicaciones) no puede dejar de sospechar que estamos tra-

4. Idem.

5. August B. Hollingshead, «Medical sociology: A brief review», Health and Society, 51 (1973): $531-542$. 
tando de un área muy subdesarrollada. La educación en sociología de la medicina concentra alguna atención, pero se debe fundamentalmente a la enseñanza de otros grupos profesionales, especialmente médicos, enfermeras, sanitarios, asistentes sociales, etc. Los sociólogos de la medicina tienen que conformarse, a menudo, con la idea de que una buena formación en sociología general debe garantizar suficientemente el desarrollo de sociólogos de la medicina competentes en su materia."

\section{Programas de sociología de la medicina}

La intención original fue limitar este trabajo a la situación de los países de la Comunidad Económica Europea, es decir: Francia, la República Federal Alemana, Italia, Bélgica, Holanda, Luxemburgo, Reino Unido, Irlanda y Dinamarca. Posteriormente se decidió prologarlo con una breve descripción sobre los Estados Unidos. Hay que tener en cuenta que la educación en ciencias de la conducta es mucho más desarrollada en los Estados Unidos. Aparte, la contribución de Mauksch ofrece la oportunidad de presentar un resumen sinóptico de los Estados Unidos. Para el caso de los países de la Comunidad Europea hicimos uso de un cuestionario por correo que fue enviado a un número de científicos en esos países. Somos conscientes de que esta primera investigación no es exhaustiva, pero representa un paso hacia ella.

\section{Estados Unidos de América}

Seguimos aquí el esquema de Mauksch, resumiendo algunas características de la educación en sociología de la medicina en los Estados Unidos. En 1972-73 el número de programas de sociología de la medicina en los Estados Unidos era al menos 25 y un total de 56 departamentos de sociología con asignaturas de sociología de la medicina. Hollingshead menciona 39 programas de sociología médica en USA y Canadá, y un total de 47 departamentos de sociología con algún tipo de enseñanza de sociología de la medicina en los USA.

En la Tabla 1 se puede ver la distribución del número de profesores en los departamentos con enseñanza de sociología de la medicina. La mayoría $(82 \%)$ de los departamentos con una asignatura tienen sólo

6. Yvo Nuyens, «Teaching medical sociology to graduate students in sociology», Conference on the Sociology of Medicine (Varsovia, 20-26 agosto 1973). 
TABLA 1

Departamentos con sociología de la medicina en USA

\begin{tabular}{ccc}
$\begin{array}{c}\text { Profesores de } \\
\text { sociología de la medicina }\end{array}$ & $\begin{array}{c}\text { Programas completos de } \\
\text { sociología de la medicina }\end{array}$ & $\begin{array}{c}\text { Asignaturas sueltas de } \\
\text { sociología de la medicina }\end{array}$ \\
\hline Ninguno & - & $2 \%$ \\
1 & $12 \%$ & 41 \\
2 & 16 & 41 \\
3 & 20 & 9 \\
4 & 28 & 4 \\
5 ó más & 24 & 4 \\
TOTAL & $100 \%$ & $100 \%$ \\
\end{tabular}

TABLA 2

Profesores de sociología de la medicina en USA

\begin{tabular}{|c|c|c|}
\hline Tipos de contratos & $\begin{array}{l}\text { Programas completos } \\
\text { de sociologia de la } \\
\text { medicina }\end{array}$ & $\begin{array}{l}\text { Asignaturas sueltas } \\
\text { de sociología de la } \\
\text { medicina }\end{array}$ \\
\hline $\begin{array}{l}\text { Profesor numerario en Departa- } \\
\text { mentos de Sociología } \\
\text { Contrato doble con otro depar- } \\
\text { tamento o Facultad de Medi- }\end{array}$ & $33 \%$ & $75 \%$ \\
\hline 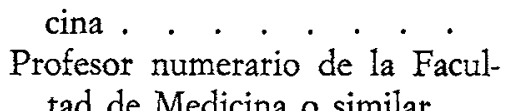 & 49 & 12 \\
\hline 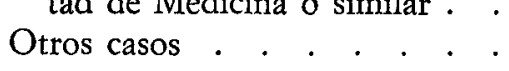 & $\begin{array}{r}2 \\
17\end{array}$ & 8 \\
\hline TOTAL . . . . . . . . . & $\begin{array}{c}100 \% \\
(89)\end{array}$ & $\begin{array}{c}100 \% \\
(91)\end{array}$ \\
\hline
\end{tabular}

uno o dos profesores que se identifican con la sociología de la medicina; mientras que sólo un $28 \%$ de los departamentos con programas de sociología de la medicina tienen esa cantidad de profesores. En la 
Tabla 2 se observa que de los 89 profesores de los 25 departamentos con programas de sociología de la medicina, la mitad (48\%) mantienen un contrato doble con una facultad de medicina. Sólo un tercio $(33 \%)$ son únicamente profesores en departamentos de sociología.

TABLA 3

Estudiantes de sociología de la medicina a nivel graduado en USA

\begin{tabular}{ccc}
\hline Número de estudiantes & $\begin{array}{c}\text { Asignaturas sueltas de } \\
\text { sociología de la medicina }\end{array}$ & $\begin{array}{c}\text { Programas completos de } \\
\text { sociología de la medicina }\end{array}$ \\
\hline Ninguno & $20 \%$ & $75 \%$ \\
$1-4$ & 12 & 16 \\
$5-9$ & 32 & 7 \\
$10-14$ & 28 & - \\
15 ó más & 8 & $100 \%$ \\
TOTAL & $100 \%$ & $(56)$ \\
\hline
\end{tabular}

La Tabla 3 muestra la distribución de estudiantes graduados en sociología de la medicina. En total hay 180 estudiantes que se especializan en sociología de la medicina en esos departamentos con programa especial. En total, el número de estudiantes en los 25 programas y 56 departamentos con otro tipo de enseñanza en sociología de la medicina es superior a 550. En los 25 programas, alrededor de 400 estudiantes siguieron un curso en sociología de la medicina (200 estudiantes siguieron un curso al menos). El resto de los 56 departamentos tuvieron alrededor de 35 estudiantes que siguieron un curso en sociología de la medicina.

\section{Bélgica}

En la Universidad Católica de Lovaina existe un programa de sociología de la medicina de dos años de duración, que empezó a funcionar en 1969. Hasta 1969 sólo había dos años de ciencias sociales que incluían algunos cursos en sociología de la medicina y otras materias relacionadas. En la actualidad, en el primer año hay un curso de sociología médica, un seminario de sociología médica y clases prácticas. En el segundo año 
hay otro seminario de sociología de la medicina, más un seminario combinado de sociología y psicología médicas.

En el curso de sociología médica se cubren los temas más relevantes de la sociología médica, en forma de introducción a la teoría y la investigación en sociología de la medicina. En el seminario de sociología médica (primer año) el temario cambia cada año. Así por ejemplo, en 1970-71 se trató del paciente mental, en 1971-72 del análisis del consumo médico, en 1972-73 de la conducta sanitaria preventiva, y en 1973-74 de las actitudes del paciente respecto del hospital. En las clases prácticas cada alumno visita (durante una semana) diversas secciones de un hospital. El seminario de sociología de la medicina (segundo año) fue introducido en 1972. Durante el primer año se discutieron los problemas del médico general en Bélgica; en 1973-74 de la comunidad terapéutica. Este seminario del segundo año es una continuación lógica del seminario del primer año. El seminario combinado de sociología y psicología médicas reúne estudiantes de sociología y de medicina, siendo opcional para ambos grupos. Los temas que se desarrollan son: muerte, aspectos sociales de la salud y la enfermedad, el modelo médico en medicina y psiquiatría, enseñanza médica y de ciencias de la conducta.

En total los estudiantes tienen 120 horas de clase en el primer año (más las clases prácticas) y 90 horas en el segundo año (más el diseño de la tesis). El programa es obligatorio, aunque según la nueva estructura (1969), ofrece a los estudiantes un alto grado de flexibilidad e independencia en la selección de asignaturas. El seminario combinado de sociología y psicología médicas, así como las clases prácticas, son opcionales.

Los cursos en sociología de la medicina tienen un examen oral final con tres temas: (a) un tema del programa, (b) una revisión crítica de un artículo seleccionado por el propio estudiante, y (c) una aplicación del temario a un problema real. Los seminarios no tienen exámenes fijos, y la actuación colectiva e individual de los estudiantes es evaluada a través de todo el curso. Al final los propios estudiantes realizan un juicio crítico individual del seminario.

En el segundo año el alumno redacta una memoria en contacto con su director de tesina. Ésta debe ser defendida ante un tribunal. Se compone aproximadamente de 100 a 150 páginas, sobre un tema libre, en general de una lista de temas que el profesor desea estimular y promover. Otros temas no incluidos en esa lista también son aceptados.

Los sistemas de enseñanza en los cursos consisten en lecciones magistrales y discusiones sobre artículos de la lista de lecturas. En los seminarios se realizan trabajos individuales y en equipo, que posteriormente se discuten. Se hacen prácticas sobre diversas técnicas de investigación. 
El departamento está formado por un catedrático (Yvo Nuyens) y cuatro adjuntos, trabajando con dedicación exclusiva.

\section{Francia}

En la Escuela de Altos Estudios de París, no dependiente de la Universidad, se da un curso de sociología de la medicina para estudiantes postgraduados. El programa empezó en 1973. La Escuela de Altos Estudios es una institución pública y nacional con carácter científico y cultural, un organismo autónomo, y entre sus objetivos está la formación de investigadores en las ciencias sociales, tendiendo a evitar todo tipo de control por parte de la universidad. No enseña a estudiantes de primer ciclo.

El curso de sociología de la medicina (que empezó en 1973) tiene como objeto el enseñar a los investigadores y estudiantes avanzados franceses las tendencias principales en investigación sociológica dentro del campo de la sanidad. También trata de comunicar a los profesionales sanitarios las dimensiones sociológicas de su práctica. El curso comprende temas como: investigación sociológica sobre la muerte, utilización de los servicios sanitarios, la organización de establecimientos sanitarios. En total se imparten 60 horas anuales de clase, en forma opcional, que se distribuyen en discusiones de lecturas y de investigaciones concretas. No hay un sistema fijo de exámenes ni tesis final. La responsabilidad del curso recae sobre Claudine Herzlich.

La sociología de la medicina también existe en el Departamento de Sociología de Nanterre en forma de seminario voluntario.

\section{Polonia}

En la Academia Polaca de Ciencias, de la Universidad de Varsovia, se imparte un curso monográfico sobre sociología de la medicina, que empezó a funcionar en 1972. El programa está todavía en desarrollo.

El estudiante de sociología de la medicina debe poseer un conocimiento específico de medicina. Debe también mantener una orientación de la sociología de la medicina, en cooperación con otras disciplinas, con una función socio-técnica precisa. Finalmente se intenta un énfasis en estudios macrosociológicos. En el contenido específico de los cursos encontramos: las características de la medicina como una ciencia práctica, el concepto médico, uso de datos médicos, el médico, la enfermera, los 
establecimientos sanitarios, el sistema sanitario, el enfermo o el paciente, problemas sanitarios, enfermedad y rehabilitación como categorías sociológicas, indicadores socio-médicos de salud, el papel del sociólogo de la medicina. La enseñanza de la sociología de la medicina está a cargo de Magdalena Sokolowska et alii.

\section{Reino Unido}

El profesor Stacey informa que hay diversos sitios en Gran Bretaña, en donde se enseña sociología de la medicina. La Universidad de Keele enseña un curso voluntario a estudiantes de primer ciclo, Leicester sociología de la sanidad, y hay un curso especial en el Swansea University Coilege. Aparte existe enseñanza de sociología de la medicina en el Bedford College y en la Universidad de Aberdeen.

El Bedford College de la Universidad de Londres tiene un programa unual de enseñanza postgraduada de sociología de la medicina, que empezó en 1969. Los objetivos principales de este curso son, primero, educar a científicos sociales en enseñanza, investigación y administración dentro del campo médico. Segundo, abrir este campo a la investigación sociológica. El temario incluye aproximadamente: análisis comparativo e histórico del cuidado médico ( 60 horas), factores sociales en la enfermedad y la respuesta a la enfermedad ( 60 horas), técnicas de investigación social: conceptos y perspectivas (60 horas), métodos estadísticos y de medida ( 30 horas), estadísticas epidemiológicas y movimiento natural de la población ( 15 horas), introducción a la medicina (15 horas), proyectos de investigación (22 horas). En total hay 262 horas aproximadamente de clase al año, y el curso es obligatorio. Los exámenes incluyen uno de tres horas sobre técnicas de investigación social, y tres temas (dos mil palabras cada uno como máximo) en cada una de estas dos áreas: organización de servicios sanitarios y factores sociales en la enfermedad. Los estudiantes reciben la opción de cinco preguntas en cada uno de los temas, y tienen cinco semanas para desarrollarlos. Aparte se supone que los estudiantes realizan una revisión crítica de la bibliografía, o un análisis de un problema específico basado en datos secundarios o de una observación sistemática personal. No se suele permitir que los estudiantes obtengan sus propios datos, debido al gasto de tiempo que esto supone. La forma de enseñanza es en forma de seminarios, en donde los estudiantes deben leer la bibliografía específica. En algunas ocasiones son los directores del seminario $\mathrm{u}$ otros conferenciantes los 
que dirigen la discusión. Entre los profesores se encuentran: M. Jefferys, G. W. Brown y S. Hiller.

En el Swansea University College hay un programa de sociología de la medicina a estudiantes de sociología del primer ciclo. El programa empezó en 1973, e incluye temas como: discusión del concepto de salud y enfermedad y sus diferencias culturales, aspectos de la sociología del cuidado sanitario, conducta enfermiza y percepción de la enfermedad, el papel del enfermo, organización de los servicios sanitarios, profesiones sanitarias, el proceso de profesionalización, procesos de interacción y transacción en los servicios sanitarios. El curso está a cargo de Margaret Stacey et alii.

En la Universidad de Aberdeen existe un programa de sociología de la medicina para estudiantes de todos los ciclos. Los estudios del primer ciclo empezaron en 1968, y los estudios graduados en 1970. Los estudios graduados están planeados para la enseñanza de métodos de investigación en la sociología de la medicina. El curso acelerado en 15 semanas abre la historia y estructura de los sistemas sanitarios, epidemiología, la organización médica desde un punto de vista interaccionista, técnicas de investigación y metodología. En el programa se incluyen lecciones magistrales, seminarios, investigación original y la presentación de una tesis. La enseñanza está a cargo de Raymond Illsey y el personal del M. R. C. Medical Sociology Unit.

\section{Otros países}

Programas de educación en sociología de la medicina no existen en Dinamarca, Alemania o Luxemburgo. En el caso de Italia e Irlanda no se pudo obtener información.?

7. En España no existen programas de educación en sociología de la medicina. Los cursos más relacionados son impartidos en la Escuela de Sanidad (a médicos especialistas en sanidad pública), o por los departamentos de psicología y psiquiatría de las facultades de medicina (a estudiantes de medicina). En la Universidad Autó. noma de Barcelona, el departamento de sociología mantiene un curso sobre la profesión psiquiátrica española (a nivel de segundo ciclo) durante el curso 1975-76. Existe también un seminario de sociología de la medicina (sobre el tema de la reforma sanitaria) en el Instituto Italiano de Cultura (en Barcelona). No existe, por ahora, ningún departamento de sociología con cursos fijos de sociología de la medicina ni para sociólogos ni para médicos, ni es posible obtener una especialización en sociología de la medicina dentro del país. (N. del T.) 


\section{Resumen}

En la Tabla 4 se presentan las características principales de la enseñanza de la sociología de la medicina en Europa. No hay suficiente información para formular conclusiones válidas más generales. La sociología de la medicina no suele estar específica o explícitamente en los programas de estudios de sociología. Tan sólo unos pocos departamentos de sociología ofrecen un programa específico de sociología de la medicina. Alli donde se desarrolla un programa o una asignatura se debe generalmente al impacto de un sociólogo que muestra un interés particular por la sociología de la medicina. Incluso para el caso de los países europeos, nosotros todavía ignoramos el número exacto de programas y asignaturas, el número de profesores, el número de estudiantes, o el número de especialistas en sociología de la medicina. Aparte hay también programas de sociología de la medicina en algunas facultades de medicina europeas.

Se observa pues una fuerte polarización entre los Estados Unidos y Europa. Mientras que en los Estados Unidos se ha alcanzado un cierto nivel de saturación, la enseñanza de la sociología de la medicina en Europa todavía es un área de especialización subdesarrollada.

\section{Preguntas y problemas}

Mientras que hasta aquí nos hemos detenido en los programas existentes, en esta sección vamos a formular algunas preguntas y problemas en torno a los requisitos didácticos y profesionales de los programas de enseñanza. La educación en sociología de la medicina no es simplemente un problema de enseñar unos cursos; es más una cuestión de ayudar a los estudiantes a adquirir información, comprensión, técnicas, actitudes, apreciación e interés, que les ayudarán a convertirse en verdaderos sociólogos de la medicina. Esto sugiere el problema de los principios pedagógicos científicos. Por otra parte, lo que significa ser "verdadero sociólogo de la medicina» no es un tema primariamente académico, sino que está determinado por la sociedad, y por lo tanto sugiere el problema de los requisitos profesionales de un programa de enseñanza de la sociología. 
Características generales de la enseñanza

\begin{tabular}{|c|c|c|c|c|c|c|c|c|}
\hline \multirow[b]{3}{*}{ País } & \multirow[b]{3}{*}{ Universidad } & \multirow[b]{3}{*}{$\begin{array}{l}\text { Sociblogos de } \\
\text { la medicina }\end{array}$} & \multirow{3}{*}{$\begin{array}{c}\text { Año ers que } \\
\text { empezo el } \\
\text { programa }\end{array}$} & \multicolumn{5}{|c|}{ Programa de estudios } \\
\hline & & & & \multicolumn{2}{|c|}{ Sociologia de la medicina } & \multicolumn{3}{|c|}{ En la Universidad } \\
\hline & & & & $\begin{array}{c}\text { Asignaturas } \\
\text { sueltas }\end{array}$ & $\begin{array}{l}\text { Programa } \\
\text { completo }\end{array}$ & $\begin{array}{c}1 e r \\
\text { ciclo }\end{array}$ & $\begin{array}{r}2 .^{\circ} \\
\text { ciclo } \\
\end{array}$ & $\begin{array}{c}3 e r \\
\text { ciclo }\end{array}$ \\
\hline \multirow[t]{2}{*}{ BÉIGICA } & Lovaina & Nuyens & 1969 & - & $\mathrm{X}$ & - & $\mathrm{X}$ & $X$ \\
\hline & Antwerp & Nuyens & 1972 & $\mathrm{X}$ & 一 & - & $\mathrm{X}$ & - \\
\hline Francia & $\begin{array}{l}\text { Ecole } \\
\text { Pratique }\end{array}$ & Herzlich & 1973 & $\mathrm{X}$ & - & - & - & - \\
\hline POLONIA & Varsovia & Sokolowska & 1972 & $X$ & - & $\mathrm{X}$ & $\mathrm{X}$ & - \\
\hline GRAN & Bedford & Jefferys & 1969 & - & $\mathrm{X}$ & - & - & $\mathrm{X}$ \\
\hline \multirow[t]{5}{*}{ BRETAÑA } & Swansea & Stacey & 1973 & $X$ & - & $\mathrm{X}$ & - & - \\
\hline & Keele & 一 & - & $\mathrm{X}$ & - & $\mathrm{X}$ & 一 & - \\
\hline & Leicester & - & - & $X$ & - & $\mathrm{X}$ & - & - \\
\hline & Aberdeen & Illsey & 1968 & $X$ & $X$ & $x$ & $X$ & $X$ \\
\hline & Sussex & Richards & 一 & $X$ & - & - & $X$ & - \\
\hline
\end{tabular}

\section{Los requisitos didácticos}

La enseñanza en sociología de la medicina se encara con requisitos didácticos y con principios pedagógicos que están inherentes en todo proceso de enseñanza y socialización. Cualquier actividad que conlleva enseñanza y aprendizaje implica inevitablemente tres componentes esenciales: estudiante, profesor y temario. En relación con estos componentes se pueden mencionar algunos principios pedagógicos.

(1) El estudiante. La educación es la planificación del cambio en la conducta de un estudiante a lo largo de un período de tiempo. Esto implica que el profesor debe tener bien claro los cambios que tienen que producirse en la conducta del estudiante para adecuarse a sus funciones y roles profesionales; esto significa que el temario tiene que ser planeado conforme a estos cambios. Aunque hay diversas teorías que ofrecen explicaciones diferentes sobre el fenómeno de aprendizaje, algunos principios son generalizables. Por ejemplo, el proceso de aprendizaje es individual;

8. J. Gallagher, «Preparing a curriculum in the social sciences for medical students», Acta Socio-Medica Scandinavica, 3 (1971): 143-150. 
a sociología de la medicina em Europa

\begin{tabular}{|c|c|c|c|c|c|c|c|c|c|}
\hline \multicolumn{3}{|c|}{ Estudiantes } & \multicolumn{4}{|c|}{ Requisitos } & \multicolumn{3}{|c|}{ Método de enseñanza } \\
\hline \multirow{2}{*}{$\begin{array}{l}\text { uera de la } \\
\text { iniversidad } \\
\end{array}$} & \multirow{2}{*}{$\begin{array}{c}\text { Del } \\
\text { departamento } \\
\text { de sociologia } \\
\end{array}$} & \multirow{2}{*}{$\begin{array}{c}\text { De otros } \\
\text { departamentos } \\
\text { y faxultades } \\
\end{array}$} & \multicolumn{2}{|c|}{ Asignatura } & \multirow{2}{*}{$\begin{array}{l}\text { Medias de } \\
\text { boras al año }\end{array}$} & \multirow{2}{*}{$\begin{array}{r}\text { Tesis c } \\
\text { tesina } \\
\end{array}$} & \multirow[b]{2}{*}{ Clase } & \multirow{2}{*}{$\begin{array}{l}\text { Semi- } \\
\text { nario } \\
\end{array}$} & \multirow{2}{*}{$\begin{array}{c}\text { Clases } \\
\text { prácticas }\end{array}$} \\
\hline & & & Obligatoria & Opcional & & & & & \\
\hline- & $X$ & - & $x$ & $x$ & 210 & $X$ & $\mathrm{X}$ & $X$ & $X$ \\
\hline - & $\mathrm{X}$ & - & - & $\mathrm{X}$ & 30 & $\mathrm{X}$ & $\mathrm{X}$ & $\mathrm{X}$ & 一 \\
\hline $\mathrm{x}$ & $\mathrm{x}$ & $\mathrm{x}$ & - & $\mathrm{x}$ & 60 & - & - & $\mathrm{X}$ & - \\
\hline - & $\mathrm{X}$ & - & - & - & - & - & $\mathrm{X}$ & - & - \\
\hline- & $\mathrm{x}$ & $\mathrm{X}$ & $\mathrm{x}$ & - & 262 & $\mathrm{x}$ & - & $\mathrm{X}$ & $\mathrm{x}$ \\
\hline- & $X$ & - & - & $\mathrm{X}$ & - & - & $x$ & $X$ & - \\
\hline- & - & - & - & $\mathrm{X}$ & - & - & - & - & - \\
\hline- & - & - & - & $X$ & - & - & - & - & - \\
\hline - & $\mathrm{X}$ & - & - & $\mathrm{X}$ & - & $X$ & $X$ & $X$ & $\mathrm{X}$ \\
\hline - & $X$ & - & - & $\mathrm{X}$ & - & - & - & $X$ & - \\
\hline
\end{tabular}

cada estudiante conlleva su propia base cognitiva y emocional y su peculiar experiencia del pasado. El aprendizaje es más eficaz cuanto más activa es la participación del estudiante. El proceso de aprendizaje que tiene más en cuenta las motivaciones del estudiante es más eficaz que el que no las tiene en cuenta. El aprendizaje que se refuerza con un sistema de recompensas es más eficaz que si no existe este sistema. La aplicación de estos principios a los programas de enseñanza de sociología de la medicina lleva a preguntarse si existen métodos específicos de enseñanza en este campo o alguna investigación sobre el tipo de motivaciones de los estudiantes en sociología de la medicina. Hasta ahora, hay muy poco conocimiento sistemático sobre estos temas.

(2) El profesor. El profesor tiene que enseñar y ayudar al estudiante a aprender. El hecho de que el profesor enseñe no correlaciona necesariamente con que el alumno aprenda. De los principios antes mencionados se pueden derivar algunas reglas para la enseñanza: (a) un método de enseñanza que individualiza el proceso de aprendizaje puede tener más éxito que otro método menos individual; (b) un método que necesita que el estudiante actúe, piense o sienta es más probable que termine con éxito que otro que no acentúa estas características; (c) un método de 
enseñanza que permita al estudiante un frecuente e inmediato feedback de su actuación es más probable que consiga éxito que un método con bajo nivel de feedback. Todas estas reglas implican un cierto nivel de técnicas pedagógicas; implican que los profesores deberían tener alguna preparación pedagógica o en metodología educacional. La pregunta que debemos hacernos es hasta qué nivel estos principios se han aplicado en los programas de sociología de la medicina.

(3) El temario. La preparación de un temario de una asignatura consiste en tres etapas lógicas. La primera es la formulación de los objetivos de la enseñanza concreta que debe quedar encuadrada en el marco más general del programa de enseñanza. Sin este conjunto de objetivos es imposible desarrollar la segunda etapa, la planificación ordenada de un programa educativo, incluso a nivel de un proceso individual de enseñanza y aprendizaje. Finalmente es necesario desarrollar un sistema para evaluar eficazmente los resultados del programa. Los objetivos pueden ser clasificados en tres categorías globales: por «conocimiento» entendemos la información que un estudiante tiene a su disposición y que utilizará en situaciones particulares; por «técnicas» nos referimos a que el estudiante puede demostrar un nivel determinado de preparación al desarrollar las actividades para las que ha sido socializado; por «actitudes» entendemos que el estudiante tiene ciertas creencias, sentimientos y tendencias de respuesta que determinan la forma en que se conduce en sus relaciones interpersonales. Los objetivos, si se hacen explícitos, se restringen generalmente a las dimensiones de conocimiento y técnicas, y simplemente pasan por alto las actitudes que hasta ahora habían sido dejadas a la intuición y buen ejemplo. Sin embargo, en el campo de la sociología de la medicina debemos preguntarnos si estas actitudes no merecen una atención especial. Sería importante considerar: el equilibrio entre investigación y políticas; la facilidad de reformular constantemente una definición médica determinada del problema en una definición sociológica; la facilidad de colaboración con otras disciplinas; la actitud a utilizar una perspectiva a largo plazo con respecto a efectos concretos del trabajo profesional producido; una actitud crítica en lo que concierne al rol y función específica del sociólogo en cuanto a la sociedad en general y el cuidado médico en particular.

\section{Requisitos profesionales}

Recientemente, y a través de una revista sociológica profesional, se desarrolló un importante debate sobre los requisitos profesionales de un 
programa de sociología en la parte flamenca de Bélgica." Aunque esta polémica se refiere a la sociología en general, es obvio que sus conclusiones generales pueden aplicarse a programas de enseñanza de sociología de la medicina. Se pueden tener en cuenta los siguientes requisitos profesionales.

(a) Pluralismo de los programas científicos. La pregunta crucial es: ¿Qué función es considerada como central por un científico? En otras palabras, este tema se refiere al concepto de actividad científica en sí misma. Desde un punto de vista internacional se pueden distinguir al menos tres programas científicos en relación con el papel profesional del sociólogo. ${ }^{\text {io }}$ Primero, un programa teórico empírico, dirigido fundamentalmente a la construcción de teorías válidas con un alto nivel de información; en otras palabras, la ampliación del conocimiento sobre una realidad dada. Segundo, un programa crítico filosófico, siguiendo la formulación y argumentación de la acción como el objetivo de la actividad científica. Tercero, un programa práctico, en el cual el objetivo de la actividad científica no es otro que la planificación de estrategias de acción sobre la base de ciertos objetivos y del conocimiento científico actual. Parece claro que éstos son programas complementarios y no competitivos o excluyentes. Toda enseñanza de la sociología debe, por lo tanto, incluir estos tres programas. En el caso de los programas de sociología de la medicina puede decirse que primariamente, si no exclusivamente, es el programa teórico empírico el que se desarrolla. Esto sucede incluso a expensas de los otros dos tipos, a pesar de que algunos autores, precisamente en conexión con los servicios sanitarios, han acentuado la necesidad de una sociología que resolucionase problemas, o lo que se ha llamado «ingeniería social» o «socio-técnica», definida por Sokolowska como «una teoría de la práctica de la acción socialmente efectiva»."

(b) Pluralismo de orientaciones teóricas. Dentro del programa empírico teórico de la ciencia, la sociología se caracteriza actualmente por una gran variedad de escuelas, paradigmas, teorías, modelos, perspectivas y enfoques, que van descle el todavía actual funcionalismo estructural, la sociología del conflicto y el neomarxismo, hasta la sociología de la acción y la etnometodología. Un programa de enseñanza de sociología

9. C. Lammers, «De professionele vereisten van een sociolog sociologische opleiding», Sociologische Gids, 2 (1973): 74-87.

10. R. Wippler, «Het pluralisme van theorieën en van werk-programma's: Eer kommentaar op Lammers», Sociologiscbe Gids, 4 (1973): 270-278.

11. M. Sokolowska, «On the scope of medical sociology» Seminar on the Teacbing of the Social Sciences in Medical Education (OMS, 1969), pp. 2-5. 
debe proveer a los estudiantes con la oportunidad de adquirir las herramientas para comparar sistemáticamente las orientaciones teóricas más importantes, con el objeto de percibir las ventajas y limitaciones de estos enfoques para resolver los diversos tipos de problemas sociales y teóricos. La sociología de la medicina ofrece en particular un abanico de orientaciones teóricas divergentes, donde los enfoques sistémico-teóricos, de conducta e interaccional se alternan constantemente. En consecuencia, estos programas de sociología de la medicina son particularmente importantes para presentar diferentes enfoques, los comparan sistemáticamente, examinan las diferencias esenciales que pueden ser observables, analizan el enfoque que tiene mayor éxito para un tipo de problemas dados y estudian hasta qué punto un enfoque implica otro.

(c) Conocimiento y berramientas de la investigación social. Dejando en el aire cuánto consenso concierne los dictados de la observación y el método científicos, se puede suponer que cada sociólogo debe estar informado de la unidad y variedad de los métodos de investigación. El estudiante no sólo debe adquirir conocimiento de estos métodos, sino además una preparación elemental en el tratamiento de este conocimiento. Aparte, podemos afirmar que la investigación del cuidado médico implica algunos problemas metodológicos específicos. Debido al fuerte desarrollo de la investigación sociológica médica, un cierto caudal de conocimiento se ha hecho disponible en estos últimos años sobre planificación y diseño, recolección de datos y análisis en la investigación sanitaria. Se puede ejemplificar esto con la publicación reciente del Milbank Memorial Fund, sobre Research Metbods in Health Care. ${ }^{12}$ Es evidente que estas publicaciones pueden jugar un papel importante en la formación profesional de los sociólogos de la medicina.

(d) Reflexiones acerca del papel y función de los sociólogos en la sociedad. Cada programa de enseñanza de la sociología, y por lo tanto de sociología de la medicina, debe proveer al estudiante con la oportunidad de reflexionar sobre la responsabilidad, papel y función de la sociología y el sociólogo en la sociedad. La sociología se encuentra aquí en una situación única, ya que los factores sociales y las consecuencias del trabajo científico son por sí mismos objetos de nuestra disciplina. El estudiante de sociología de la medicina debe, por lo tanto, conocer esto, y la forma en que la ciencia influencia la vida social y a su vez es producto de todo tipo de fuerzas sociales. Es precisamente el sociólogo que trabaja en el campo de la sanidad el que es más apto para enfrentarse con una serie de problemas de valores, como fue señalado recientemente

12. J. McKinlay (ed.), Research Metbods in Healtb Care (Nueva York: Milbank Memorial Fund, 1973). 
por Freeman, Levine y Reeder. También Zola ha resaltado esta relación. ${ }^{13}$ Consideramos que este tipo de reflexión debe ser integrada en el programa de enseñanza.

\section{Conclusiones}

El punto de partida de este estudio fue la observación de que el nivel de especialización y la tasa de profesionalización de una disciplina son altamente dependientes de una formación educativa en este campo especial. Por esta razón la primera parte del artículo describe el status actual de la enseñanza de la sociología de la medicina en Europa y los Estados Unidos. En ese análisis se puso de manifiesto una gran diferencia en el nivel de desarrollo entre los USA y los países europeos. La segunda parte de este artículo analiza los requisitos didácticos y profesionales de los programas de sociología de la medicina. Se puede afirmar que ambos aspectos han sido infravalorados $y$ poco desarrollados en la organización de la sociología de la medicina como una disciplina científica. Sin embargo, es precisamente la sociología de la medicina la que ofrece excelentes oportunidades para producir contribuciones en estas áreas, dentro de la sociología.

Lisbeth M. CLAus

Department of Sociology

St. Louis University

St. Louis, Missouri 63103

USA

\section{Yvo NUYENS}

Sociologisch Onderzoeksinstituut

Edw. Van Evenstraat 2B

Katholieke Universiteit Leuven

Bélgica

13. I. Zola, De medische macht (Boom: Meppel, 1973). 